\title{
A New Approach to Design Smith Predictor Based Fractional Order Controllers
}

\author{
Khosro Khandani and Ali Akbar Jalali
}

\begin{abstract}
In this paper a new approach to design and analyze Smith predictors with fractional $P I^{\lambda} D^{\mu}$ controllers for time delay systems is presented. Since the fractional controller has been used in Smith predictor scheme, delay component can be eliminated from the closed-loop characteristic equation which is of fractional order. Simulation results demonstrate the successful performance of the proposed controller. Stability analysis of the closed-loop system in time domain shows the validity of the results.
\end{abstract}

Index Terms- fractional order controller, Smith predictor.

\section{INTRODUCTION}

Dead times, or time delays, are found in many processes in industry. Dead times are mainly caused by the time required to transport mass, energy or information, but they can also be caused by processing time or by the accumulation of time lags in a number of simple dynamic systems connected in series[1]. Compared to processes without delays, the presence of a delay in the process greatly complicates the analytical aspects of the control system design, making it more difficult to achieve a satisfactory level of control [2]. Time-delay has been a common phenomenon to overcome whenever we close a feedback loop for the purpose of controlling any system. Recent increase of control applications in the variety gives more importance to systematic methods to cope with time delay. In order to compensate the negative effects of time delay, a well-known and highly effective dead-time compensator for stable processes is Smith predictor [3]. In this predictor scheme, a mathematical model of the process is implemented in an internal feedback loop around a conventional controller. The distinctive scheme was proposed by O.J.M.Smith approximately 50 years ago, and is still attracting much attention for its usefulness. The major advantage of the Smith predictor is that delay issues can be ignored when designing the controller.

Fractional-order dynamic systems and controllers, which are based on fractional-order calculus have been gaining attention in several research communities since the last few years[4]. A few recent works in this direction as well as

Manuscript received September, 23, 2009

Khosro Khandani is with the Electrical Engineering Department, Iran University of Science and Technology (IUST), Tehran, Iran (phone: +98-9144172814 ; e-mail: khosro.khandani@gmail.com).

Ali A. Jalali was with West Virginia University, USA. He is now with the Department of Electrical Engineering, Iran University of Science and Technology, Tehran, Iran (e-mail: ajalali@iust.ac.ir). schemes for digital and hardware realizations of such systems can be traced in [5], and [6]. In frequency domain Vinagre et al. proposed an approach based on expected crossover frequency and phase margin for the controller design[7]. Using the concept of fractional derivatives and integrals Podlubny proposed fractional-order $\mathrm{PI}^{\lambda} D^{\mu}$ controllers which is an extension of proportional-integral-derivative (PID) controllers that have been used for several decades in industries for process control applications. Podlubny demonstrated the effectiveness of such controllers for actuating the responses of fractional-order systems[8]. In fractional-order proportional-integral-derivative controllers, I and D operations are usually of fractional order; therefore, besides setting the proportional, derivative and integral constants $K_{p}, K_{d}, K_{i}$, we have two more parameters: the order of fractional integration $\lambda$ and that of fractional derivative $\mu$ [4]. In this paper, the fractional order PID controller is used in a Smith predictor scheme to control plants with time delays. In [9] a frequency domain design approach for time delay systems has been proposed. The fractional controller in the Smith predictor structure in [9] is designed to fulfill stability conditions in frequency domain. The proposed methodology in [9] has been applied to design controllers for water distribution in a main irrigation canal pool.

By using Smith predictor, delay component is removed from the closed loop characteristic equation. In this paper, stability of the closed loop system which is of fractional order is analyzed in time domain. Stable region is expanded from the left half plane to the right half plane, and the closed loop system will not necessarily be unstable even if the poles fall on the right half plane. Two time delay systems, one with a long and the other with a short time delay are considered as design examples and their responses to unit step input are simulated.

\section{Fractional CAlculus and Fractional Control}

Fractional calculus is a 300-year-old topic. The theory of fractional order derivatives was developed mainly in the 19th century. However, applying fractional order calculus to dynamic systems control is just a recent focus of interest [8]. Fractional-order dynamic systems were studied only marginally in the design and practice of control systems in the last few decades. However, in the recent years, emergence of effective methods in differentiation and integration of non-integer order equations makes fractional-order systems more and more attractive for the 
systems control community. The TID controller [10], the CRONE controllers [11],[12] and [13] and the fractional lead-lag compensator [14] and [15] are some of the well-known fractional-order controllers. In some of these papers it is verified that the fractional-order controllers can have better disturbance rejection ratios and less sensitivity to plant parameter variations compared to the traditional controllers [16]. The ${ }^{2} I^{\lambda} D^{\mu}$ controller was proposed as a generalization of the controller with integrator of real order $\lambda$ and differentiator of real order $\mu$ [17].

\section{A. Definitions}

Fractional calculus is a generalization of integration and differentiation to non-integer (fractional) order fundamental operators represented as ${ }_{a} D_{t}^{\lambda}$, where $a$ and $t$ are the limits and $\lambda(\lambda \in R)$ the order of the operation. This operator is a notation for taking both the fractional derivative and the fractional integral in a single expression. For positive $\lambda$ it denotes derivative and for negative $\lambda$ it denotes integral actions.

From a purely mathematical point of view, there are several ways to define fractional-order derivatives and integrals. The commonly used definitions for fractional derivatives are Grunwald-Letnikov, Riemann-Liouville and Caputo's definitions [8]. The definition for Caputo's fractional derivative of order $\lambda$ with respect to the variable $t$ goes as follows:

${ }_{0} D_{t}^{\lambda} f(t)=\left\{\begin{array}{c}\frac{1}{\Gamma(m-\lambda)} \int_{0}^{t} \frac{f^{m}(\tau) d \tau}{(t-\tau)^{\lambda+1-m}}, m-1<\lambda<m \\ \frac{d^{m}}{d t^{m}} f(t), \lambda=m\end{array}\right.$

where $m$ is the first integer larger than $\lambda$, and $\Gamma(Z)$ is Euler's Gamma function. The Laplace transform of the Caputo's fractional derivative is:

$$
\begin{aligned}
& L\left\{{ }_{0} D_{t}^{\lambda} f(t)\right\}=s^{\lambda} F(s)-\sum_{k=0}^{n-1} s^{\lambda-1-k} f^{(k)}(0), \\
& n-1<\lambda \leq n \in N .
\end{aligned}
$$

One distinct advantage of using Caputo's definition is that, only integer order derivatives of function $f(t)$ appear in the Laplace transform of the Caputo's fractional derivative[16]. For zero initial conditions, (2) reduces to:

$$
L\left\{{ }_{0} D_{t}^{\lambda} f(t)\right\}=s^{\lambda} F(s) \text {. }
$$

\section{B. Continuous Time Approximations}

Direct implementation of fractional-order transfer functions is problematic. Hence, to implement these transfer functions, integer order approximations of the fractional transfer functions are determined. There are many different methods to find such approximations. Charef [18], Oustaloup [19], Carlson [20] and Matsuda [21] approximations are the well-known approximations of the fractional-order transfer functions. From the control theoretic point of view, the proposed methods are divided into two groups. Methods that use continued fraction expansions (CFE) and interpolation techniques such as Carlson and Matsuda methods, and methods that use curve fitting or identification techniques such as Oustaloup and Charef methods[22].

Since none of the existing methods transcend others regarding to all desires, it is not possible to say that which one is the best [23]. A comprehensive comparison of these approximation methods has been given in Chapter 3 of [23]. In the numerical simulations of the present work, the Oustaloup method is used to find rational approximation of the fractional operators. This simple method provides a continuous approximation of fractional transfer functions using recursive allocation of zeros and poles to achieve an admissible accuracy. The approximated transfer function based on Oustaloup method is determined using the following definition:

$$
s^{v} \approx k \prod_{n=1}^{N} \frac{1+s / \omega_{z, n}}{1+s / \omega_{p, n}}, \quad 0<v<1
$$

Gain $k$ is adjusted so that both sides of (4) have unit gain at $1 \mathrm{rad} / \mathrm{s}$. The number of poles and zeros of the approximated transfer function $(N)$ and the frequency range $\left(\left[\omega_{l}, \omega_{h}\right]\right)$ are selected beforehand. $\omega_{z, n}$ and $\omega_{p, n}$ are calculated by the following equations:

$$
\begin{gathered}
\omega_{z, 1}=\omega_{l} \sqrt{\eta}, \quad \omega_{p, n}=\omega_{z, n}\left(\frac{\omega_{h}}{\omega_{l}}\right)^{v / N}, n=1,2, \ldots, N \\
\omega_{z, n}=\omega_{p, n-1}\left(\frac{\omega_{h}}{\omega_{l}}\right)^{(1-v) / N}, n=2,3, \ldots, N
\end{gathered}
$$

For $-1<v<0$, the approximated transfer function is determined by inverting (4). To find the approximation for $|v|>1$, first $s^{v}$ is written in the form $s^{v}=s^{[v]} s^{\delta}$ and then the term $s^{\delta}$ is replaced by the approximation given in (4). The extension of the approximation strongly depends on the number of poles and zeros $(\mathrm{N})$. The lower values of $\mathrm{N}$ result in simpler models, however, ripples are appeared both in gain and phase plots. Such ripples would be disappeared by increasing $\mathrm{N}$ in the expense of having computationally more complex models[24]. Also, the approximation is reliable only in the frequency range $\left[\omega_{l}, \omega_{h}\right]$. It is desirable to widen the approximating frequency range as much as possible, but increase in the width of the frequency range reduces the approximating accuracy.

\section{SMITH PREDICTOR CONTROL}

The Smith predictor is widely used for the control of systems with time delays. Fig. 1 presents a block diagram of the conventional Smith predictor configuration, in which $\mathrm{L}$ is the dead time of the process, $\mathrm{C}(\mathrm{s})$ denotes the controller, and $\mathrm{P}(\mathrm{s})$ is the delay free part of the plant, which is assumed to be open-loop stable. The transfer function from the reference input $\mathrm{r}$ to the output $\mathrm{y}$ of the system has the form:

$$
\frac{Y(s)}{R(s)}=\frac{C(s) P(s) e^{-L s}}{1+C(s) P(s)}
$$

As shown in (7), the main advantage of the Smith predictor is that the delay component can be eliminated from the 
characteristic equation for the closed-loop system. In this paper $\mathrm{C}(\mathrm{s})$ is a fractional controller, and the characteristic equation would be a polynomial of ' $s$ ' with fractional powers.

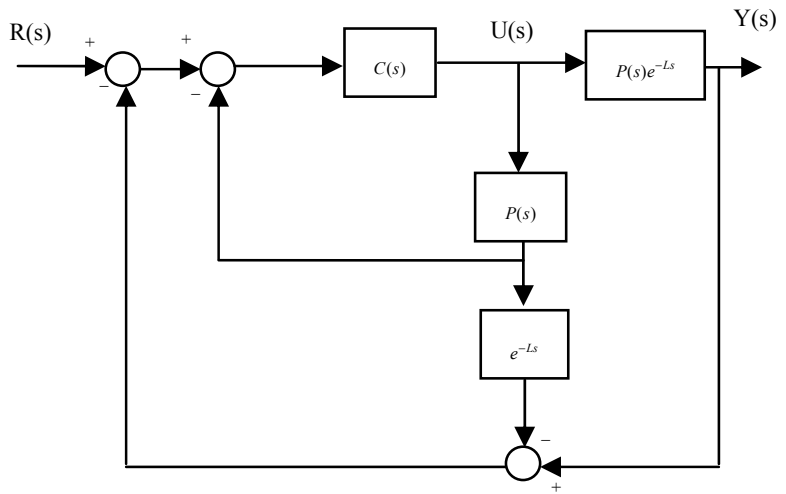

Figure1 Smith predictor scheme

\section{STABILITY ANALYSIS}

In general linear time-invariant fractional order systems can be described by the following transfer function of non-commensurate real orders [9] of the following form:

$$
G(s)=\frac{b_{m} s^{\beta_{m}}+\ldots+b_{1} s^{\beta_{1}}+b_{0} s^{\beta_{0}}}{a_{n} s^{\alpha_{n}}+\ldots+a_{1} s^{\alpha_{1}}+a_{0} s^{\alpha_{0}}}=\frac{Q\left(s^{\beta_{k}}\right)}{P\left(s^{\alpha_{k}}\right)}
$$

Where $\alpha_{k}$ and $\beta_{k}(\mathrm{k}=0,1,2, \ldots)$ are real numbers. It is well-known that an integer order LTI system is stable if the roots of the characteristic polynomial $P(s)$ are negative or have negative real parts if they are complex conjugate. This means that they are located on the left of the imaginary axis of the complex s-plane. When dealing fractional order systems, it is important to bear in mind that $P\left(s^{\alpha}\right), \alpha \in R$ is a multi-valued function of $\mathrm{s}$, the domain of which can be viewed as a Reiman surface (see e.g. [25]). For checking the stability of $P\left(s^{\alpha}\right), \alpha \in R$ the existing stability test methods for dynamic systems with integer orders such as Routh table technique cannot be directly applied. This is due to the fact that the characteristic equation of the LTI fractional order system is in general not a polynomial but a pseudo-polynomial function of the fractional powers of $s$.

In the particular case of commensurate order systems, it holds that $\alpha_{k}=\alpha k, \beta_{k}=\alpha k,(0<\alpha<1), \forall k \in Z$, and the transfer function has the following form:

$$
G(s)=K_{0} \frac{\sum_{k=0}^{M} b_{k}\left(s^{\alpha}\right)^{k}}{\sum_{k=0}^{N} a_{k}\left(s^{\alpha}\right)^{k}}=K_{0} \frac{Q\left(s^{\alpha}\right)}{P\left(s^{\alpha}\right)}
$$

With $\mathrm{N}>\mathrm{M}$ the function $\mathrm{G}(\mathrm{s})$ becomes a proper rational function in the complex variable $s^{\alpha}$ and can be expanded in partial fractions of the form:

$$
G(s)=K_{0}\left[\sum_{i=1}^{N} \frac{A_{i}}{s^{\alpha}+\lambda_{i}}\right],
$$

where $\lambda_{i},(\mathrm{i}=1,2, . ., \mathrm{N})$ are the roots of the polynomial $P\left(s^{\alpha}\right)$ or the system poles that are in general complex conjugate. Stability condition can then be stated that [26], [27]:

A commensurate order system described by a rational transfer function (9) is stable if $\left|\arg \left(\lambda_{i}\right)\right|>\alpha \frac{\pi}{2}$, with $\lambda_{i}$ the i-th root of $P\left(s^{\alpha}\right)$.

Stability region on s-plane is depicted in Fig.2. It can be observed that the stability region covers a percentage of the right half plane, and unlike the integer order case, stability region is not the left side of the imaginary axis.

Since the delay component that is a hard-to-deal-with element in stability analysis in time domain, can be removed from the characteristic equation of the closed-loop system, stability of the transfer function from $\mathrm{Y}(\mathrm{s})$ to $\mathrm{R}(\mathrm{s})$ in Fig.1 can be directly verified based on the stability condition mentioned above.

\section{CASE StUdy}

To demonstrate the capabilities of the proposed approach, two processes are considered in the following examples. For the first process a $P I^{\lambda}$ controller is used in the Smith predictor structure, and in the second example the $P I^{\lambda}$ controller is replaced by a $P I^{\lambda} D^{\mu}$ controller.

\section{A. Example 1}

A stable second order plus dead time process is adopted. This process was considered by Takahara et al.[28]. The simulation conditions given are the same as in the literature [28]. In [28] the transfer function of the process is

$$
G(s)=\frac{0.04936 s+0.00197}{s^{2}+0.0401 s+0.000169} e^{-0.3 s},
$$

The fractional $P I^{\lambda}$ controller $\mathrm{C}(\mathrm{s})$ in Smith predictor scheme in Fig. 1 is:

$$
C(s)=4+\frac{40}{s^{0.4}},
$$

In this example and the following one, the fractional controller is approximated with integer order transfer functions. As mentioned before the approximated transfer function is determined based on Oustaloup method. The parameters in (5) and (6) are selected as follows:

$\omega_{l}=0.01, \omega_{h}=1000, N=5$,

Characteristic equation of the closed-loop system according to (9) will be:

$s^{2.4}+0.2375 s^{1.4}+1.974 s+0.0081 s^{0.4}+0.079$,

Assuming $\alpha=0.2$ in (9), the characteristic equation above can be rewritten as a polynomial of the complex variable $s^{\alpha}$ as: 
$P\left(s^{\alpha}\right)=\left(s^{\alpha}\right)^{12}+0.2375\left(s^{\alpha}\right)^{7}+1.974\left(s^{\alpha}\right)^{5}+0.0081\left(s^{\alpha}\right)^{2}+0.079$.

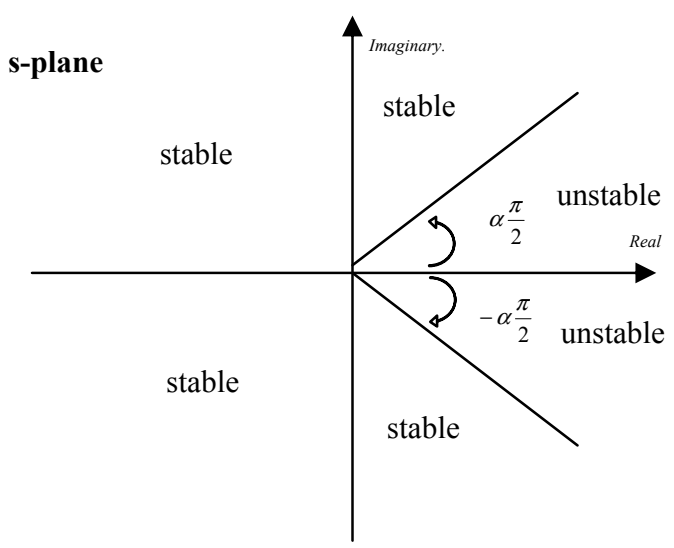

Figure2 Stability region

The roots of polynomial $P\left(s^{\alpha}\right)$ are:

$$
\begin{aligned}
& \lambda_{1}=-1.208, \lambda_{2}=-1.1208, \lambda_{3,4}=-0.1622 \pm j 0.4996 \\
& \lambda_{5,6}=-0.6699 \pm j 0.8702, \lambda_{7,8}=0.9974 \pm j 0.4965 \\
& \lambda_{9,10}=0.4250 \pm j 0.3089, \lambda_{11,12}=0.2329 \pm j 1.0592
\end{aligned}
$$

Since the first six roots have negative real parts, it is evident that they satisfy the stability condition discussed earlier. We apply the stability condition to the remaining six roots. If $\left|\arg \left(\lambda_{i}\right)\right|>\frac{\pi}{10} \operatorname{rad}(18 \mathrm{deg})$, with $\lambda_{i}$ the i-th root of $P\left(s^{\alpha}\right)$, the closed-loop system is stable. We have:

$\left|\arg \left(\lambda_{7,8}\right)\right|=26.4773,\left|\arg \left(\lambda_{9,10}\right)\right|=36.0288,\left|\arg \left(\lambda_{11,12}\right)\right|=77.6383$

They all satisfy the condition, Therefore, stability of the closed-loop system is corroborated. Step response of the system and the control signal are shown in Fig.3 and Fig.4.

\section{B. Example 2}

Following the same procedure as in example 1, we consider a water level control problem. The approximated first order

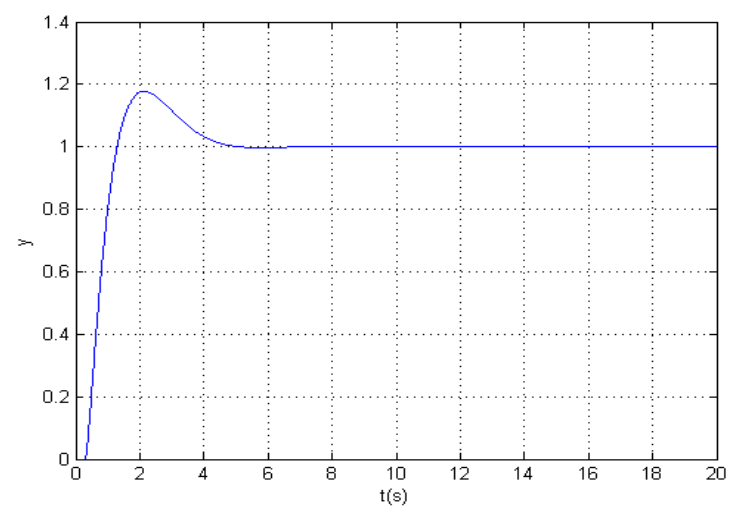

Figure3 Step response of the system in example 1

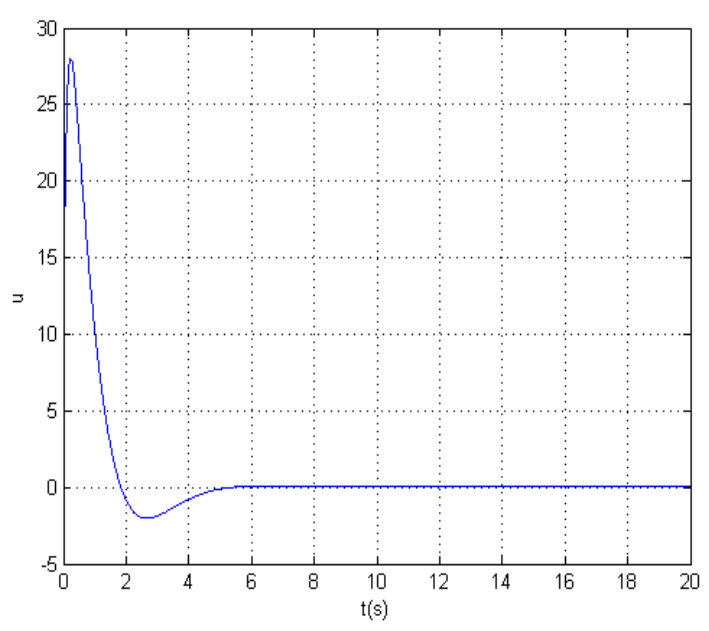

Figure4 Control signal in example 1

SISO coupled tank system was obtained in [29] as:

$$
G(s)=\frac{2.06}{21.1528 s+1} e^{-20.0472 s}
$$

The controller $\mathrm{C}(\mathrm{s})$ in Fig. 1 is assumed to be a $P I^{\lambda} D^{\mu}$ controller as follows:

$$
C(s)=7.5+2 s^{0.1}+\frac{5}{s^{0.7}}
$$

Step response of the system with this controller in Smith predictor scheme and the control attempt are shown in Fig.5 and Fig.6.

Stability of the closed-loop system can be analyzed by obtaining the characteristic equation. Replacing $\mathrm{C}(\mathrm{s})$ the controller in (7) by the $P I^{\lambda} D^{\mu}$ controller above, we obtain the characteristic equation as follows:

$21.1528 s^{1.7}+4.12 s^{0.8}+16.45 s^{0.7}+10.3$

And $P\left(s^{\alpha}\right)$ in (9) with $\alpha=0.1$ will equal:

$P\left(s^{\alpha}\right)=$

$P\left(s^{0.1}\right)=21.1528\left(s^{0.1}\right)^{17}+4.12\left(s^{0.1}\right)^{8}+16.45\left(s^{0.1}\right)^{7}+10.3$

The roots of this equation are:

$\lambda_{1}=-0.9058, \lambda_{2,3}=-0.0581 \pm j 1.01, \lambda_{4,5}=-0.4749 \pm j 0.8143$

$\lambda_{6,7}=-0.6591 \pm j 0.6806, \lambda_{8,9}=-0.9413 \pm j 0.3347$

$\lambda_{10,11}=0.7957 \pm j 0.4204, \lambda_{12,13}=0.6006 \pm j 0.8307$

$\lambda_{14,15}=0.2315 \pm j 0.8559, \lambda_{16,17}=0.9583 \pm j 0.26$

According to Fig. 2 and with $\alpha=0.1$, Stability region of the system has extended to the right half plane and covers a significant percentage of it, as shown in Fig.7. With such a wide stable region, it is much easier to design a stabilizing controller that overall closed-loop system satisfies stability conditions. It is clear that none of the roots of the system fall in the unstable region, which is shown in Fig.7. hence, stability of the closed-loop system is verified. 


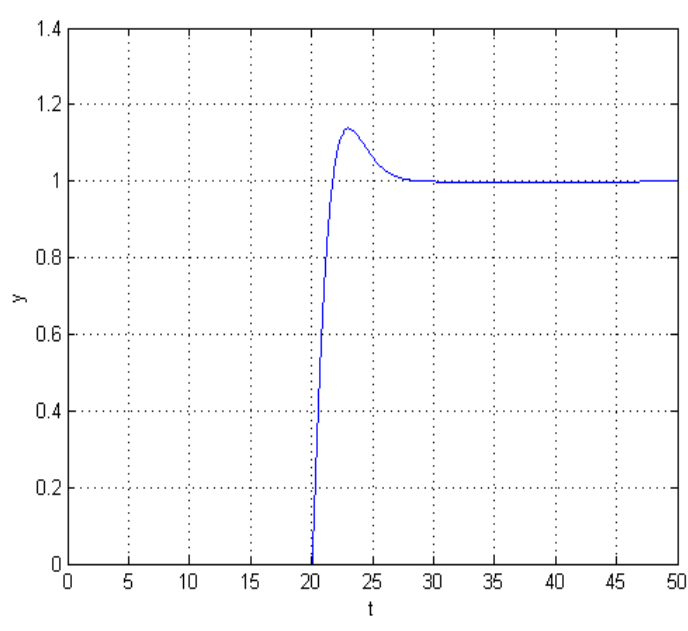

Figure5 step response of the system in example 2

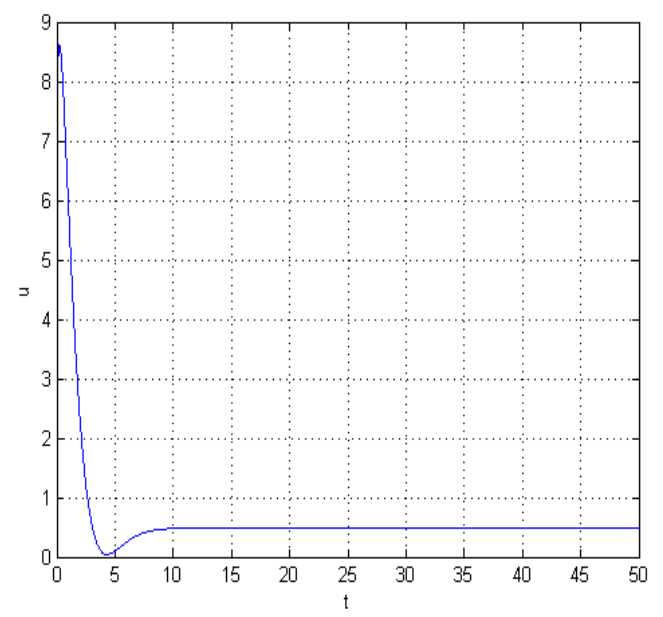

Figure6 Control signal in example 2

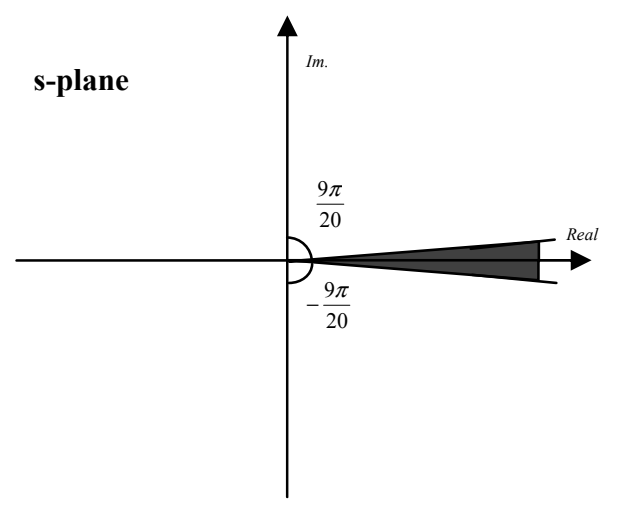

Figure7 Unstable region shown in grey

\section{ACKNOWLEDGMENT}

The authors would like to express their sincere thanks to Mr. Iraj Kheirizad from Electronic Research Center of Iran University of Science and Technology, and also Dr. Mohammad Saleh Tavazoei from Electrical Engineering Department at Sharif University of Technology, Tehran, Iran.

\section{REFERENCES}

[1] J.E.Normey-Rico, E.F.Camacho, Control of dead-time processes, Springer-Verlag, London, 2007.

[2] Y.D. Chen , P.C. Tung, C.C. Fuh, "Modified Smith predictor scheme for periodic disturbance reduction in linear delay systems," Journal of process control, vol.17, 2007, pp. 799-804.

[3] O.J. Smith, “A controller to overcome dead time," ISA Journal, vol.6, 1959, pp. 28-33.

[4] Biswas , S. Das , A. Abraham, S. Dasgupta, "Design of fractional-order $P I^{\lambda} D^{\mu}$ controllers with an improved differential evolution," Engineering applications of artificial intelligence, vol. 22, 2009, pp. 343-350.

[5] Y.Q. Chen, H. Ahn, D. Xue, "Robust controllability of interval fractional order linear time invariant systems,"Proceedings of the ASME 2005 International Design Engineering Technical Conferences \& Computers and Information in Engineering Conference, Long Beach, CA, 2005, pp. 1-9.

[6] Y.Q. Chen, D. Xue, H. Dou, "Fractional calculus and biomimetic control," Proceedings of the First IEEE International Conference on Robotics and Biomimetics (RoBio04), Shengyang, China, 2004.

[7] B.M.Vinagre, I. Podlubny, L. Dorcak, V.Feliu, "On fractional PID controllers: a frequency domain approach" Proceedings of IFAC Workshop on Digital Control-PID, Spain, 2000.

[8] I. Podlubny, Fractional Differential Equations, Academic Press, San Diego,USA, 1999.

[9] V. Feliu-Batlle , R.R. Perez , F.J.C. Garcia , L.S. Rodriguez, "Smith predictor based robust fractional order control: Application to water distribution in a main irrigation canal pool," Journal of Process Control, vol. 19, 2009, pp. 506-519.

[10] B.J. Lune, Three-parameter tunable tilt-integral derivative (TID) controller, US Patent US5371 670, 1994.

[11] A. Oustaloup, J. Sabatier, P. Lanusse, "From fractal robustness to CRONE control," Fractional Calculus and Applied Analysis, vol. 1, no. 2, 1999, pp.1-30.

[12] A. Oustaloup, X. Moreau and M. Nouillant, "The CRONE suspension," Control Engineering Practice, vol. 4, no. 8, 1996, pp. 1101-1108.

[13] A. Oustaloup, B. Mathieu and P. Lanusse, "The CRONE control of resonant plants: application to a flexible transmission," European Journal of Control, vol.1, no. 2 , 1995, pp. 113-121.

[14] H.F. Raynaud, A. Zergainoh, "State-space representation for fractional order controllers," Automatica, vol.36, 2000, pp. 1017-1021.

[15] C.A. Monje, V. Feliu, "The fractional-order lead compensator," IEEE International Conference on Computational Cybernetics, Vienna, Austria, September, 2004

[16] M.S.Tavazoei, M.Haeri, "Chaos control via a simple fractional-order controller," Physics Letters A, vol. 372, 2008, pp.798-807.

[17] I. Podlubny, "Fractional-order systems and $P I^{\lambda} D^{\mu}$ controllers," IEEE Transactions on automatic control, vol. 44, no. 1, 1999, pp. 208-214.

[18] A. Charef, "Analogue realisation of fractional-order integrator, differentiator and fractional $P I^{\lambda} D^{\mu}$ Controller," IEE Proc. Control Theor. Appl. , vol.153, no.6, 2006, pp. 714-720.

[19] A. Oustaloup, F. Levron, F. Nanot, B. Mathieu, "Frequency band complex non-integer differentiator: characterization and synthesis," IEEE Trans. Circuits Systems I: Fundam. Theory Application, vol.47, no1, 2000, pp. 25-40.

[20] G.E. Carlson, C.A. Halijak, "Approximation of fractional capacitors by a regular newton process," IEEE transactions on Circuit Theory, vol.11, no. 2,1964 , pp. $210-213$.

[21] K. Matsuda, H. Fujii, “ $H^{\infty}$ optimized wave absorbing contro: analytical and experimental results," Journal of guidance, control and dynamics, vol.16, no.6, 1993, pp.1146-1153.

[22] B.M. Vinagre, I. Podlubny, A. Hernandez, V. Feliu, "Some approximations of fractional order operators used in control theory and applications ," Fractional calculus and applied analysis, no.3, vol.3, 2000, pp. 231-248.

[23] D. Valerio, Fractional robust system control, $\mathrm{PhD}$ thesis, Instituto Superior Técnico, Lisboa, 2005.

[24] D. Valerio, J. Sá da Costa, "Tuning of fractional PID controllers with Ziegler-Nichols-type rules," Journal of Signal Processing, vol. 86, no.10, 2006, pp. 2771-2784.

[25] D. A. Pierre, "Minimum Mean-Square-Error design of distributed parameter control systems," ISA Transactions, vol. 5, 1966, pp. 263-271. 
[26] D. Matignon, "Stability result on fractional differential equations with applications to control processing," In: IMACS - SMC Proceeding, July, Lille, France, 1996, pp. 963-968.

[27] D. Matignon, "Stability properties for generalized fractional differential systems," Proceeding of Fractional Differential Systems: Models, Methods and Applications, vol. 5, 1998, pp. 145-158.

[28] T. Takehara, T. Kunitake, H. Hashimoto, F. Harashima, "The control for the disturbance in the system with time delay," International Workshop on Advanced Motion Control, AMC 1, 1996, pp. 349-353.

[29] V. Bhambhani and Y.Q. Chen, "Experimental Study of Fractional Order Proportional Integral (FOPI) Controller for Water Level Control," Proceedings of the 47th IEEE Conference on Decision and Control, Cancun, Mexico, 2008, pp.1791-1796.

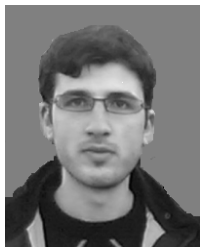

Khosro Khandani was born in Tabriz, Iran, in 1984. He received his B.Sc. degree in Electrical Engineering from Sahand University of Technology, Tabriz, Iran, in 2007. He is now an M.Sc. student at Iran University of Science and Technology, Tehran, Iran. His research interests include fractional-order control, artificial intelligence and intelligent control, robust control, and time delay systems.E-mail: khosro.khandani@gmail.com

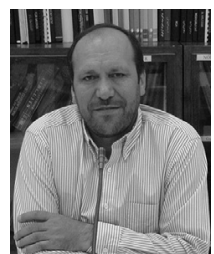

Ali Akbar Jalali was born in Damghan, Iran, in 1954 He received his B.Sc. degree in Electronics Engineering from Khajeh Nasiredin Toosi University of Technology, Tehran, Iran, May 1985. He obtained his M.Sc. degree in Electrical Engineering from Oklahama university, Norman, US, in 1988. He sequentially earned his Ph.D. and Post Doctoral in Electrical Engineering, from West 1994 respectively.

Virginia University, Morgantown, US, in 1993 and

Dr. Jalali joined the Lane Department of Computer Science and Electrical Engineering, Collage of Engineering and Mineral Resources, West Virginia University, as an adjunct Professor in 2002. His current research opportunity has been provided, in part, by a sabbatical leave from the Department of Electrical Engineering, Iran University of Science and Technology (IUST) where he has been since 1994 and he is working in Electronic Research Center, at IUST as an associate professor. His research has focused mainly in the field of Extended Kalman Filtering, Robust Control and H-infinity. Additionally, study of Information Technology and its applications like: Virtual Reality, Virtual Learning, Internet City, Rural ICT developments and Designing ICT Strategic Plan in different levels are his other research interests.Email: ajalali@iust.ac.ir 